\title{
HEALTHY AND SUSTAINABLE FOOD HABITS IN TIMES OF THE COVID-19 PANDEMIC
}

\author{
Montaña Cámara Hurtado \\ Departamento de Nutrición y Ciencia de los Alimentos. Facultad de Farmacia, Universidad Complutense de Madrid.
}

corresponding author: mcamara@ucm.es

\section{REVISIÓN}

\section{RESUMEN}

El primer reto al que nos enfrentamos en este siglo XXI es el de poder alimentar a todos los habitantes de este planeta con alimentos seguros y saludables y además de forma sostenible. Existen sociedades donde el principal problema es la disponibilidad de alimentos, lo cual deriva en malnutrición y enfermedades de la pobreza (principalmente transmisibles) junto a otras donde la disponibilidad de alimentos no es el problema, pero paradóicamente se dan casos de malnutrición por malas elecciones dietéticas que derivan en las denominadas enfermedades de la riqueza (no transmisibles), como es el caso de las enfermedades cardiovasculares como principal causa de mortalidad. La irrupción a principios del año 2020 de la emergencia provocada por el coronavirus SARS-Cov2 ha hecho de esta situación un nuevo reto en todo el mundo, sin distinción de países con ingresos altos o bajos. Este discurso pretende abordar la importancia de mantener un adecuado estatus nutricional para mantener un buen estado de salud, minimizar los efectos negativos provocados por la COVID-19 así como facilitar la recuperación de los pacientes. Se revisará la evidencia científica relativa al papel de los micronutrientes y compuestos bioactivos en el fortalecimiento del sistema inmune, un efecto beneficioso sinérgico basado en sus mecanismos de acción complementarios. Se tratará además el nuevo concepto de "dieta de salud planetaria" que considera la relevancia de las elecciones dietéticas, por su implicación tanto para la salud humana como para el medio ambiente, manteniendo los recursos disponibles: agua tierra y suelo. El objetivo final es profundizar en el conocimiento científico de manera que pueda ser de utilidad para la sociedad; la promoción de la seguridad alimentaria, mediante una alimentación saludable y sostenible.

\section{ABSTRACT}

The first challenge we face in this 21 st century is to be able to feed all the inhabitants of this planet with safe and healthy food in a sustainable way. There are societies where the main problem is the availability of food, which leads to malnutrition and diseases of poverty (mainly communicable). In other societies, the availability of food is not the problem, but paradoxically there are cases of malnutrition due to poor dietary choices, which lead to the so-called wealth diseases (non-communicable), such as cardiovascular diseases as the main cause of mortality. The emergence at the beginning of 2020 of the emergency caused by the SARS-Cov2 coronavirus has made this situation a new challenge throughout the world, without distinction of countries with high or low income. The speech aims to address the importance of maintaining an adequate nutritional status to maintain good health, minimize the negative effects caused by COVID-19 as well as facilitate the recovery of patients. Scientific evidence regarding the role of micronutrients and bioactive compounds in strengthening the immune system will be reviewed, a synergistic beneficial effect based on their complementary mechanisms of action. It will also discuss the new concept of "planetary health diet" that considers the relevance of dietary choices, due to their implication for both human health and the environment, while maintaining available resources: water, land and soil. The ultimate goal is to deepen scientific knowledge so that it can be of use to society; promoting food security through healthy and sustainable eating.

\section{Palabras Clave: \\ Alimentación saludable Antioxidantes \\ Sistema inmune Micronutrientes Alimentación sostenible \\ Keywords: \\ Healthy food Antioxidants Immune system Micronutrients Sustainable food systems}




\section{INTRODUCCIÓN}

El primer reto al que al que nos enfrentamos en este siglo XXI es el de poder alimentar a todos los habitantes de este planeta con alimentos seguros y saludables de forma sostenible. Varios informes internacionales nos han hecho reflexionar sobre las consecuencias que tienen, el modo de producir alimentos, así como nuestras elecciones dietéticas, tanto para nuestra salud individual como para el medio ambiente donde habitamos (1,2). La carga de la malnutrición en todas sus formas sigue constituyendo un desafío y es probable que la seguridad alimentaria y el estado nutricional de los grupos de población más vulnerables se deterioren aún más debido a las repercusiones socioeconómicas y sanitarias de la pandemia COVID-19.

En la actualidad coexisten dos realidades en cuanto a la alimentación: las dos caras de la Seguridad Alimentaria (Food Safety vs Food Security). Existen sociedades donde el principal problema es la disponibilidad de alimentos, lo cual deriva en malnutrición y enfermedades de la pobreza (principalmente transmisibles) junto a otras donde la disponibilidad de alimentos no es el problema, pero paradóicamente se dan casos de malnutrición por malas elecciones dietéticas que derivan en las denominadas enfermedades de la riqueza, generalmente enfermedades no transmisibles (3). Entre ellas, las cardiopatías son desde hace 20 años la causa principal de mortalidad en todo el mundo, si bien en la actualidad provocan más muertes que nunca. Por otro lado, las enfermedades infecciosas han constituido una de las principales causas de muerte para la especie humana desde sus orígenes. En 2019, la neumonía y otras infecciones de las vías respiratorias inferiores fueron el grupo más mortífero de enfermedades transmisibles siendo la causa principal de muerte en países de ingresos bajos (4).

La irrupción a principios del año 2020 de la emergencia provocada por el coronavirus SARS-Cov2 causante de la pandemia COVID-19 ha hecho de esta situación un auténtico reto siglo del XXI en todo el mundo, sin distinción de países con ingresos altos o bajos.

En la lucha contra las enfermedades causadas por virus la medida más eficaz para su control es el desarrollo de vacunas que cumpla con las garantías de calidad, seguridad y eficacia (5). En este sentido, la base de datos de seguimiento y panorama de vacunas candidatas COVID-19, de la OMS, compila información detallada y actualizada sobre las vacunas candidatas COVID-19 en desarrollo (6).

La enfermedad COVID-19, está caracterizada por provocar un síndrome respiratorio agudo grave, que cursa con sintomatología algo diferente en función del grupo de población afectada, y se ob- serva que aquellos con su sistema inmunitario comprometido, pueden correr un riesgo más elevado de sufrir complicaciones graves, de forma similar a lo que se observa con otras enfermedades respiratorias. Pero no debemos olvidar otros factores de riesgo que condicionan la severidad de la enfermedad, como es el estatus nutricional.

\section{INFLUENCIA DE LOS FACTORES DIETÉTICOS EN LA CARGA GLOBAL DE ENFERMEDAD}

La dieta subóptima es un importante factor de riesgo prevenible de las enfermedades no transmisibles (ENT). Este hecho ha sido confirmado por los resultados del estudio Global Burden of Disease Study 2019 (7) tras evaluar el consumo de los principales alimentos y aporte de nutrientes en 195 países y cuantificar el impacto de su ingesta subóptima en la mortalidad y morbilidad de las ENT. Los factores dietéticos más críticos son la ingesta de granos enteros, frutas, hortalizas, semillas y legumbres entre otros por su contenido en fibra. Estos datos acaban de ser corroborados por el estudio de la American Hearth Association (8) que establece que el consumo de productos vegetales "Ayuda a reducir el riesgo de padecer numerosas enfermedades crónicas que son las principales causas de muerte, como las cardiovasculares o el cáncer". El menor riesgo de mortalidad se observó para un consumo de aproximadamente 5 porciones diarias de productos vegetales, en concreto: 2 de frutas y 3 de verduras, si bien una ingesta superior no se acompañó de una mayor disminución del riesgo (9).

Esta situación es especialmente preocupante en España, pues pese a ser un país productor de frutas y hortalizas de alta calidad, en la actualidad en España el consumo de productos vegetales, tanto en la población infantil y adolescente, según datos encuesta ENALIA, como en la población adulta mayores y embarazadas, ENALIA 2, se encuentra por debajo de la recomendación de $400 \mathrm{~g} /$ día establecida por la OMS $(10,11)$. Datos corroborados por el Informe de la Fundación Española de Nutrición en 2018 (12).

Los alimentos de origen vegetal son una fuente importante de micronutrientes y compuestos bioactivos con propiedades antioxidantes como son la vitamina C (en su forma de ácido ascórbico y dehidroascórbico), la vitamina $\mathrm{E}$ (siendo la forma más activa el alfa-tocoferol), los carotenoides (con actividad como provitamina A como el beta-caroteno, o bien con otras funciones como el licopeno, los compuestos fenólicos (como los de las familias de flavonoides, flavonoles, antocianos...), y otras sustancias cuyo consumo tiene una clara incidencia positiva en nuestra salud ) (13-15). Todos estos compuestos los podemos encontrar en frutos como los cítricos 


\section{ANALES}

(16) y hortalizas tanto cultivadas como silvestres (17), así como en cereales y legumbres (18). Todos ellos característicos de nuestra dieta mediterránea.

\subsection{Micronutrientes y estimulación del sistema inmune}

Ante nuestro objetivo de reforzar la función inmune, el primer escollo con el que nos encontramos es la enorme complejidad de este proceso y la multitud de factores involucrados, que hace que no siempre esté claro el efecto de los componentes de los alimentos sobre sus mecanismos.

El seguimiento de unas pautas dietéticas inadecuadas podría facilitar la infección o empeorar su evolución. Esto se ha visto en personas con sobrepeso y obesidad, convirtiéndose en un factor de riesgo de enfermedad grave de COVID-19. Es por ello que desde el comienzo de la pandemia distintos investigadores se han ocupado en analizar cómo una alimentación saludable, en el marco de una dieta variada y equilibrada, puede contribuir a mantener un mejor estado de salud, incluyendo un mejor funcionamiento del sistema inmune (19-22).

Los micronutrientes, como las vitaminas $A, D, C, E$ y el mineral zinc, son necesarios para garantizar la integridad estructural y funcional de la piel y membranas mucosas, que tiene un efecto barrera y representan la primera línea de defensa contra patógenos invasores (21). Los procesos de inmunidad innata, como la proliferación y diferenciación celular, entre otros procesos, depende de cantidades adecuadas de vitaminas $A, D, C, E, B_{6}$, y $B_{12}$, folato, y minerales como hierro, zinc, cobre, selenio y magnesio. Del mismo modo, la respuesta química, como es la activación del sistema del complemento y la liberación de citocinas proinflamatorias, requiere de la acción de ciertas vitaminas y minerales (en particular, vitaminas $A$, D y C, zinc, hierro y selenio). La respuesta inflamatoria cierra la brecha entre la inmunidad innata y adaptativa, y está regulada por las vitaminas $A, C, E, y B_{6}$, así como hierro, zinc y cobre. Del mismo modo, la respuesta inmune adaptativa, que incluye aquella mediada por células y la inmunidad humoral, depende nuevamente de la presencia de una variedad de micronutrientes en todas las etapas (proliferación, diferenciación y función de linfocitos, e inmunidad mediada por células). Al mismo tiempo, los micronutrientes participan en la autoprotección de las células inmunitarias (a través de mecanismos antioxidantes, por ejemplo, vitaminas $C$ y $E$, zinc, hierro, magnesio, cobre y selenio), acciones inhibidoras (vitaminas $D, B_{6}$ y) y eliminación de células agostadas mediante apoptosis y aclaramiento (lo que limita el daño tisular), acción realizada por la vitamina $C(20,21)$.

Otros compuestos bioactivos, como los ácidos grasos poliinsaturados de la serie omega-3 (ácidos $\alpha$-linolénico, eicosapentaenoico y docosahexaenoico) pueden estimular el sistema inmunitario por diferentes mecanismos. Por ejemplo, mejorando la proliferación y la actividad de las células B o disminuyendo la producción de citoquinas y de prostaglandinas proinflamatorias (19). Muchas bacterias ácido-lácticas que forman parte de la microbiota intestinal, como son los lactobacilos y bifidobacterias, pueden competir con otros microorganismos patógenos a nivel del colon, modulando además la respuesta inmune por diferentes mecanismos (23).

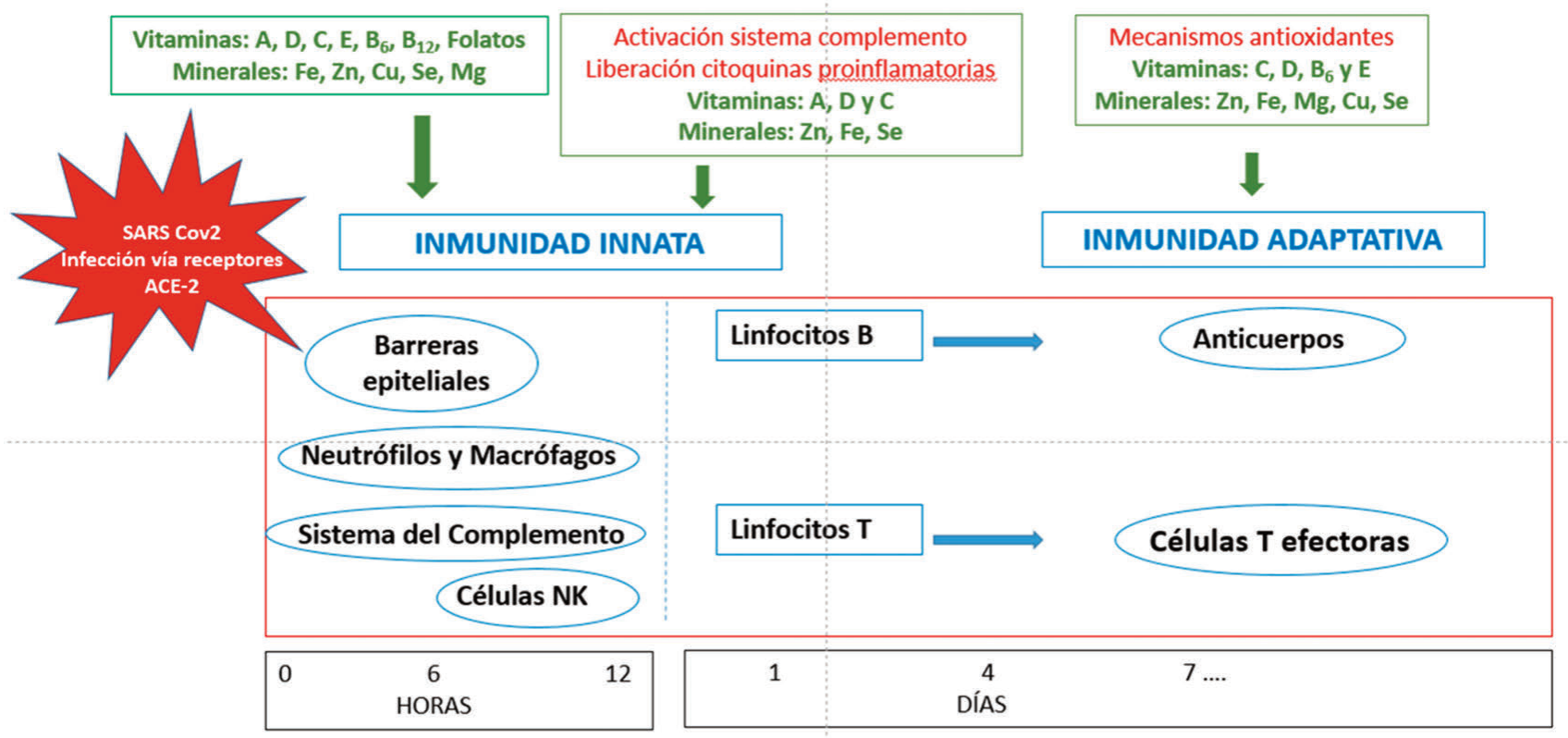

Tiempo tras la infección

Figura 1.- Relación de los micronutrientes con la respuesta inmune innata y adaptativa. Basado en: 20,21 
Por tanto, cada etapa de la respuesta inmune depende de la presencia de ciertos micronutrientes y compuestos bioactivos, que producen un efecto beneficioso sinérgico basado en sus mecanismos de acción complementarios, como se puede observar en la Figural. Un mecanismo importante es la actividad antioxidante que presentan determinados alimentos, por su composición en nutrientes y compuestos bioactivos. Esta actividad antioxidante permite reducir los niveles de la enzima convertidora de angiotensina-2 (ACE2), la cual se ha considerado un receptor en las superficies celulares para el SARS-CoV-2 (21).

De todos los micronutrientes anteriormente mencionados, la vitamina $\mathrm{D}$ es la que ha demostrado más efectos positivos contra la enfermedad COVID-19, ya que se asocia tanto con una disminución de las tasas de infección como con mejores resultados para los pacientes. La vitamina $D$ es reconocida por su papel en la regulación del metabolismo del calcio y el fosfato, así como en el mantenimiento de la mineralización ósea. También se considera como hormona inmunomoduladora. La vitamina $\mathrm{D}$ mejora la barrera física contra los virus, estimula la producción de péptidos antimicrobianos, y activa a células defensivas como los macrófagos que podrían destruir el SARS-CoV-2. Puede prevenir las tormentas de citoquinas al disminuir la producción de citoquinas inflamatorias $(24,25)$ y mantener la integridad endotelial. Además, la vitamina $D$ es un regulador clave del sistema renina-angiotensina (enzima convertidora de angiotensina 2, ACE2), que es aprovechado por el SARS-CoV-2 para entrar en las células huésped $(26,27)$.

Debe tenerse en cuenta que actualmente varios estudios epidemiológicos destacan la deficiencia de vitamina $D$ en la población general, ya sea por baja exposición solar o por una dieta baja en grasas, siendo niños pequeños, ancianos y personas obesas, los grupos poblacionales más susceptibles a sufrir esta hipovitaminosis (28), lo que les haría también más vulnerables para esta enfermedad.

Para validar las evidencias científicas encontradas hasta el momento actual, se encuentran en curso diferentes ensayos clínicos que evalúan si el uso de complementos alimenticios (incluyendo vitamina $C, D$ y $Z n$ ) puede reducir el riesgo de infección con síndrome respiratorio agudo severo, o reducir el riesgo de hospitalización, morbilidad y / o mortalidad en participantes diagnosticados con COVID-19. De ellos, solo hay un ensayo clínico terminado (NCT: 04810949) realizado (durante 6 meses) en 41 pacientes con niveles séricos de vitamina $D+/-20 \mathrm{ng} / \mathrm{ml}$. Se trata de un ensayo clínico aleatorizado comparativo que evaluó el efecto de 2 tratamientos en el personal de salud del Hospital Clínica Nova (México). El estudio finalizó el pasado 1 de marzo de 2021, y los resultados aún no se han publicado (29). Todos estos estudios en marcha son prometedores, pero aún no son concluyentes.

\section{EVIDENCIA CIENTÍFICA - DECLARACIONES DE PROPIEDA- DES SALUDABLES}

Como marco legal y certificación de la evidencia científica haremos referencia a las Declaraciones de Propiedades Saludables autorizadas por la Comisión Europea al tener un dictamen científico favorable de la Autoridad Europea de Seguridad Alimentaria (EFSA) de acuerdo con el Reglamento 1924/2006 (30,31).

Tabla 1.- Dedaraciones de propiedades saludables autorizadas por EFSA en relación a la protección del daño oxidativo y la estimulación de la función inmune.

\section{PROTECCIÓN DEL DAÑO OXIDATIVO}

Contribuye a lo profección del ADN, profef́nas y lipidos del daño oxidativo

\begin{tabular}{|c|c|}
\hline Vitamina C & Cobre \\
\hline Vitamina B2 & Manganeso \\
\hline Vitamina E & Selenio \\
\hline & Zinc \\
\hline
\end{tabular}

ESTIMULACIÓN DE LA FUNCIÓN INMUNE

Contribuye a la función normal del sistema inmune

\begin{tabular}{|c|c|}
\hline Vitamina A & Cobre \\
\hline Vitamina $\mathrm{B}_{6}$ & Hierro \\
\hline Vitamina $\mathrm{B}_{9}$ (ác. Fólico) & Selenio \\
\hline Vitamina $\mathrm{B}_{12}$ & Zinc \\
\hline Vitamina D & \\
\hline
\end{tabular}

A yuda al mantenimiento del sistema inmune como parte de

las defensas del organismo

Vitamina C

Contribuye al mantenimiento del normal fu nciona miento

del sistema inmune durante y fras la realización de ejercicio fisico intenso

Vitamina C

Contribuye a la función normal del sistema inmune en niños

Vitamina D

Basado en: EU Register on nutrition and health claims (32).

INR = Ingestas Nutricionales de Referencia; Al: Adequate intake (aporte satisfactorio). 


\section{ANALES}

RANF

Www.analesranf.com

Tabla 2.- Alimentos que pueden ser considerados como fuente de los micronutrientes para los cuales se ha autorizado alguna Declaración de Salud en relación a su contribución al normal funcionamiento del sistema inmune.

\begin{tabular}{|c|c|c|}
\hline Micronutriente & $\begin{array}{l}\text { Ingesta Nutriente Referencia } \\
\text { (INR) / Aporte satisfactorio (AI) }\end{array}$ & $\begin{array}{c}\text { Alimentos que constituyen sus fuentes } \\
\text { naturales más abundantes }\end{array}$ \\
\hline \multicolumn{3}{|c|}{ VITAMINAS } \\
\hline $\begin{array}{l}\text { Vitamin Aycurtenoides, } \\
\text { como equivalentes de } \\
\text { retinol }\end{array}$ & INR: $600-750 \mu \mathrm{g} / \mathrm{dí} 0$ & $\begin{array}{l}\text { Huevos. Hígado (cerdo, vocuno). } \\
\text { Hortalizos (espinocos, zonahorios, colobozo, boniotos) } \\
\text { Lócteos (quesos, mantequillo) }\end{array}$ \\
\hline Vitamina $B_{6}$ & INR: 1,2- $1.7 \mathrm{mg} / \mathrm{dí} 0$ & $\begin{array}{l}\text { Frutos secos (pistacho, pipas de girosol, nuez) } \\
\text { Legumbres (lentejo, cocohuete) } \\
\text { Pescados (sordino, boquerón, solmón, polometo, } \\
\text { cobollo) } \\
\text { Cornes (cordero); hígado (cerdo, vocuno) }\end{array}$ \\
\hline $\begin{array}{l}\text { Vitamina By (ácido folico y } \\
\text { derivados) }\end{array}$ & INR: $270-330 \mu \mathrm{g} / \mathrm{d}$ ío & $\begin{array}{l}\text { Hígado (cerdo, pollo). } \\
\text { Legumbres (sojo, olubios, lentejo, habos, gorbanzo) } \\
\text { Frutos secos (cocohuete) } \\
\text { Verduras (espinoco, ocelgo, espórrago verde, } \\
\text { cebollino, col rizodo, endivio, puerro, escorolo) }\end{array}$ \\
\hline Vifamina $B_{12}$ & INR: $2,2-2.4 \mu \mathrm{g} / \mathrm{dí} 0$ & $\begin{array}{l}\text { Huevos y lócteos (quesos) } \\
\text { Pescados y moriscos } \\
\text { Cornes y vísceros }\end{array}$ \\
\hline Vitamina C & INR: $60-75 \mathrm{mg} / \mathrm{dí} 0$ & $\begin{array}{l}\text { Frutos (kiwi, cítricos, freso, piño, frambueso, grosello, } \\
\text { papayo, guayoba) y hortalizos (pimiento, especies del } \\
\text { género Brossico), preferiblemente en estado crudo. }\end{array}$ \\
\hline Vitamina D & INR: 10 - 12,5 $\mu \mathrm{g} / \mathrm{dí} 0$ & $\begin{array}{l}\text { Carnes (cordero, cobrito) y vísceros } \\
\text { Huevos y lócteos (mantequillo) } \\
\text { Pescados y mariscos }\end{array}$ \\
\hline \multicolumn{3}{|c|}{ ELEMENTOS MINERALES } \\
\hline Cobre & INR: 1 - 1,3 mg/día & $\begin{array}{l}\text { Frutos secos (olmendros) } \\
\text { Legumbres (olubio, hobos, lentejo, sojo) } \\
\text { Cereoles integroles (oveno, trigo, orroz) y sus } \\
\text { derivodos }\end{array}$ \\
\hline Hierro & Al: 9 - $18 \mathrm{mg} / \mathrm{dí}$ & $\begin{array}{l}\text { Cornes y derivodos (vocuno, cordero, ovestruz, hígodo, } \\
\text { morcillo) } \\
\text { Pescados (sordinos, bocoloo) y moriscos (olmejos, } \\
\text { berberechos, langostinos, colomor, mejillón). } \\
\text { Legumbres (lentejo, olubio, sojo, hobo) } \\
\text { Frutos secos (pistocho, sésamo) }\end{array}$ \\
\hline Selenio & INR: 45 - $70 \mu \mathrm{g} / \mathrm{dí}$ & $\begin{array}{l}\text { Cornes (cerdo, ovestruz) y vísceros. Huevos } \\
\text { Cereoles integroles (trigo, centeno) y sus derivados } \\
\text { Pescodos y mariscos } \\
\text { Frutos secos (onocordo, sésomo, pipos de girosol) }\end{array}$ \\
\hline Zinc & INR: 8 - 11 mg/día & $\begin{array}{l}\text { Cereales integrales (trigo, oveno) y sus derivodos } \\
\text { Legumbres (oltramuz, soja) } \\
\text { Frutos secos (pipas de girosol, pipos de colobozo, } \\
\text { sésamo) }\end{array}$ \\
\hline
\end{tabular}

Basado en: (22,32-35) 
Respecto a las propiedades antioxidantes es preciso decir que solo siete micronutrientes (vitaminas y minerales) han mostrado suficiente grado de evidencia para poder alegar sus propiedades beneficiosas en relación a la protección del daño producido por el estrés oxidativo sobre el ADN, proteínas y grasas (32). Por otro lado, diez micronutrientes (vitaminas y minerales) han mostrado con suficiente grado de evidencia contribuir al correcto funcionamiento del sistema inmune si se consumen en cantidades suficientes para cubrir los requerimientos nutricionales diarios (32). La actividad antioxidante la ejercen por distintos mecanismos, captación de radicales libres, prevenir la propagación de la peroxidación lipídica e inhibir la inflamación.

En la Tabla 1 se muestran las declaraciones de propiedades saludables autorizadas por EFSA en relación a la protección del daño oxidativo y la estimulación de la función inmune hasta la fecha. Hay que incidir, en que el papel beneficioso derivado de la ingesta de estos micronutrientes es una contribución a dichas funciones, no una garantía de inmunidad.

\subsection{Alimentos como fuente de micronutrientes antioxi- dantes y estimulantes de la función inmune}

No hay un único grupo de alimentos que contenga todos los compuestos beneficiosos e involucrados en la mejora de la función inmune, sino que es una dieta variada y equilibrada lo que hace que todos los compuestos involucrados en dichas funciones puedan actuar de forma sinérgica. En la Tabla 2, se muestran los alimentos fuente de los micronutrientes para los que se ha autorizado la declaración de salud "contribuye al normal funcionamiento del sistema inmune", así como las ingestas de referencia para la población española de acuerdo con AESAN, 2019 (33). Para la consideración de "fuente" de un determinado micronutriente se hace referencia al cumplimiento de los requisitos del Reglamento $1169 / 2011$, contenido mínimo de un 15\% de los valores de referencia de nutrientes para cada caso concreto (34).
En algunos casos los requerimientos de los micronutrientes anteriormente mencionados no se pueden cubrir a través de la ingesta de alimentos y por ello puede ser necesario el uso adecuado de complementos alimenticios (36).

Hay que recordar que no existen complementos alimenticios que eviten la infección por ningún tipo de virus u otro agente patógeno, y, por lo tanto, no puede haber ningún producto en el mercado con tales declaraciones. Pese a ello, las autoridades europeas han observado que cada vez más productos vendidos a través de Internet se anuncian alegando tener un efecto positivo en el sistema inmunológico o que protegen contra la infección por coronavirus (37). Hasta ahora, la evidencia científica no respalda ninguna afirmación de que cualquier alimento 0 complemento alimenticio proteja contra la enfermedad COVID-19 (38).

La Sociedad Internacional de Inmunutrición (ISIIN), recomienda, en personas de edad avanzada, el aumento de la ingesta de nutrientes que han demostrado mejorar la inmunidad de las células T y B (anticuerpos), como son vitamina C, D y E, así como el mineral $Z n$, en cantidades muy superiores a sus requerimientos, como puede observarse en la Tabla 3 (38). La suplementación con Vitamina $D$ en pacientes hospitalizados también es recomendada por la Sociedad Española de Geriatría y Gerontología para mejorar el equilibrio inmunitario y prevenir la tormenta de citoquinas hiperinflamatorias (40).

\section{ALIMENTACIÓN SOSTENIBLE}

Un Sistema Alimentario Sostenible es un sistema alimentario capaz de proporcionar seguridad alimentaria y nutrición para toda la población, de manera que no se comprometan los mecanismos económicos, sociales y ambientales para que las generaciones futuras puedan contar con alimentos nutritivos en cantidad suficiente, dentro del concepto de economía circular (42).

Tabla 3.- Requerimientos nutricionales de micronutrientes: vitaminas C, D, E, y mineral Zn, establecidas por distintos organismos, en comparación con las recomendaciones ISIN para personas de edad avanzada en situación COVID.

\begin{tabular}{|c|c|c|c|}
\hline Micronutriente & ISIN, 2020 & AESAN, 2019 & EFSA, 2017 \\
\hline Vitamin C & $200 \mathrm{mg}-2 \mathrm{~g} /$ día & INR: $60-75 \mathrm{mg} /$ día & PRI: 70-110 mg/día \\
\hline Vitamin D & $10 \mu \mathrm{g}-100 \mu \mathrm{g} /$ día & INR: $10-12,5 \mu \mathrm{g} /$ día & Al: $15 \mu \mathrm{g} /$ día \\
\hline Vitamin E & $134 \mathrm{mg}-800 \mathrm{mg} /$ día & INR: $11-13 \mathrm{mg} /$ día & Al: $11-13 \mathrm{mg} /$ día \\
\hline Zinc & $30 \mathrm{mg}-220 \mathrm{mg} /$ día & INR: $8-11 \mathrm{mg} /$ día & PRI: $7,4-11,7 \mathrm{mg} /$ día \\
\hline
\end{tabular}

Basado en: 33,39,41.

INR = Ingestas Nutricionales de Referencia; PRI: Population Reference Intake (referencia nutricional para población); Al: Adequate intake (aporte satisfactorio) 
Una alimentación saludable y sostenible es un modelo de alimentación que tiene como objetivo afrontar tanto las preocupaciones de salud como las ambientales asociadas a la producción y consumo de alimentos. La pandemia de COVID-19 en la que estamos aún inmersos ha supuesto una amenaza global sin precedentes que ha alterado la vida de miles de millones de personas alrededor del planeta y ha puesto en peligro la economía global (43). A finales de 2019 todos estábamos muy concienciados de la importancia de cuidar nuestro entorno. El preservar la salud humana en tiempos de la COVID-19 ha eclipsado estas preocupaciones que, es momento de retomar. Hay que definir estrategias para el periodo post-COVID-19 que permitan retomar la senda de crecimiento sostenible (44).

Los cambios en la dieta no solo pueden mejorar la salud y prevenir enfermedades; también pueden ayudar a reducir la huella medioambiental, disminuir el uso de recursos naturales y contribuir al cuidado del medio ambiente. En este contexto surge el nuevo concepto de "dieta de salud planetaria", que considera la relevancia de las elecciones dietéticas, por su implicación tanto para la salud humana como para el medio ambiente, manteniendo los recursos disponibles: agua, tierra y suelo (45). Se estima que el duplicar la ingesta de frutas, verduras, legumbres y nueces, y reducir el consumo de carnes rojas y procesadas y azúcares añadidos al menos a la mitad, se pueden llegar a evitar más de 10 millones de muertes por año, lo que supondría una reducción de $19-23.6 \%$ en las cifras actuales de mortalidad (46). Coincide que los alimentos de origen vegetal son los que menor impacto medioambiental negativo producen medido en términos de: emisión de gases de efecto invernadero, requerimientos de uso de tierra y energía, así como un menor impacto en la acidificación y eutrofización de los suelos.

Se debe por tanto potenciar el consumo de productos vegetales. No es necesario llegar a imponer dietas veganas estrictas que pueden llevar asociados déficits nutricionales, sino abogar por dietas "flexitarianas", 0 de manera general por dietas en las que todos los alimentos están presentes pero los productos vegetales son los predominantes (47) Además, son los productos vegetales los que actualmente sufren los niveles más altos de pérdida y desperdicio, cuestión que debe ser reducida al máximo. Esta necesidad ha llevado a Naciones Unidas a declarar el 2021 como el año internacional de la frutas y verduras (48). Durante este año los países pondrán énfasis en fomentar dietas más saludables y ricas en frutas y verduras, que además de ser sostenibles y amigables con la lucha contra el cambio climático.

El conseguir establecer un sistema agroalimentario sostenible permitirá la consecución de los objetivos fijados por Naciones Unidas en la Declaración de Roma (2015): Promover sistemas alimentarios sostenibles mediante la formulación de políiticas públicas coherentes desde la producción hasta el consumo y en todos los sectores pertinentes; así como los establecidos en la Estrategia Europea FOOD $2030(49,50)$.

\section{CONCLUSIONES}

El mantenimiento de los sistemas defensivos del organismo permite afrontar mejor las situaciones de riesgo, como las derivadas de la actual pandemia COVID-19, además de seguir unas pautas higiénicas adecuadas, y potenciar la vacunación, debemos cuidar el estatus nutricional con especial incidencia en los siguientes micronutrientes con efecto antioxidante y protector del sistema inmune: vitaminas $\left(A, D, C, E, B_{6}, B_{12}\right.$ y folatos) y minerales (hierro, zinc, cobre, selenio y magnesio).

Hoy en día ya no es suficiente que los alimentos sean seguros para la población, cuestión claramente indiscutible, es necesario además que sean respetuosos con el planeta, nuestro hábitat que debemos preservar. Deben ser accesibles a todos en cualquier región del mundo, minimizando las desigualdades siendo adecuados a cada etapa y circunstancia de la vida incluso aquellas imprevistas como la pandemia COVID-19.

El conocimiento científico debe ser de utilidad para la sociedad. En este sentido, los profesionales de la salud debemos trabajar juntos potenciando en la población una alimentación saludable y sostenible, es decir, aquella que nos permite mantener un buen estado de salud, utilizando adecuadamente los recursos disponibles y contribuyendo, por tanto, a mantener el ecosistema.

\section{AGRADECIMIENTOS}

Mi más profundo agradecimiento a la Sección 5 de la RANF por haber apoyado mi candidatura a formar parte de esta Corporación y a la Junta de Gobierno por haberlo ratificado. La Real Academia de Farmacia ha formado parte de toda mi vida profesional. Aquí recibí mi primer Premio de investigación. He colaborado en publicaciones con distintos Académicos y he asistido a multitud de conferencias, todas ellas de gran valor. A ella pertenecen, $y$ han pertenecido, mis más ilustres profesores, y entre todos ellos el Profesor Dr. D. César Nombela Cano un referente científico y humano y quien hoy me presenta. El Dr. Nombela fue mi profesor de Microbiología, ya en el periodo postdoctoral siguió mis pasos en la andadura americana. En 2013 me dio la gran oportunidad de incorporarme al Claustro de la Universidad Internacional Menéndez Pelayo (UIMP) donde me permitió realizar tareas de Gestión Universitaria, una experiencia que supuso un salto cualitativo en mi desarrollo personal y profesional. Gracias César por tus palabras, por tu generosidad, por todas tus enseñanzas y lo más valioso, tu amistad; y por acompañarme en este nuevo reto. Gracias a la Presidenta de la Sección 5 por haberme permitido contextualizar mi discurso a la situación ahora a todos nos preocupa y ocupa, la pandemia COVID-19.

Agradezco muy sinceramente a todos aquellos que a lo largo de mi vida personal y profesional me han apoyado y acom- 
pañado hasta llegar hoy aquí. A mi familia, soy hija de padres farmacéuticos que me han transmitido la pasión y respeto por esta profesión, y han apoyado todas mis iniciativas. A mis amigos, la familia elegida, que siempre me han acompañado.

En el ámbito profesional, a las Doctoras Esperanza Torija y Carmen Díez, mis directoras de Tesis Doctoral, el primer gran proyecto de todo investigador. A mis compañeros de Departamento, y a muchos amigos profesores de la Facultad de Farmacia de la UCM, mi casa. A la familia UIMP, magníficos profesionales y amigos, que de la mano del Rector Nombela y Lora Tamayo hemos trabajado juntos por un bien común, la excelencia académica. Al Dr. Charles Shoemaker de la Universidad de California, que ha hecho del Campus de Davis mi segunda casa. Y muy especialmente a mi grupo de investigación, a mis compañeras con las que comparto mi día a día $y$ a todos los doctorandos de los que tanto he aprendido y seguimos creciendo juntos.

A todos, muchas gracias.

\section{REFERENCIAS}

1. FAO. El estado de la seguridad alimentaria y nutrición en el mundo. SOFI2020. Disponible en: (fao.org/publications/sofi/2020/es/).

2. IPCC. Climate change and land. Grupo Intergubernamental de Expertos sobre el Cambio Climático, 2019. Disponible en: (ipcc.ch/srccl/).

3. OMS. Principales causas de muerte y discapacidad en el mundo: 2000-2019. Disponible en: (who.int/es/news/item/09-12-2020who-reveals-leading-causes-of-death-and-disability-worldwide2000-2019)

4. FAO. Hambre e inseguridad alimentaria. Disponible en: (fao.org/hunger/es/).

5. AEMPS. Vacunas contra la COVID-19. Disponible en: (aemps.gob.es/la-aemps/ultima-informacion-de-la-aemps-acercadel-covid\%E2\%80\%9119/vacunas-contra-lacovid\%E2\%80\%9119/).

6. WHO. Draft landscape and tracker of COVID-19 candidate vaccines. Disponible en:

(who.int/publications/m/item/draft-landscape-of-covid-19-candidate-vaccines).

7. GBD Diet Collaborators. Health effects of dietary risks in 195 countries, 1990-2017: a systematic analysis for the Global Burden of Disease Study 2017. Lancet 2019; 11, 393(10184):1958-1972. doi: 10.1016/S0140-6736(19)30041-8.

8. Wang DD, Li Y, Bhupathiraju SN, Rosner BA, Sun Q, Giovannucci EL, Rimm EB, Manson JE, Willett WC, Stampfer MJ, Hu FB. Fruit and Vegetable Intake and Mortality: Results From 2 Prospective Cohort Studies of US Men and Women and a Meta-Analysis of 26 Cohort Studies. Circulation 2021; 1:43 00-00. doi: 10.1161/CIRCULATIONAHA. 120.048996

9. Wang DD, Li Y, Bhupathiraju SN, Rosner BA, Sun Q, Giovannucci EL, Rimm EB, Manson JE, Willett WC, Stampfer MJ, Hu FB. Fruit and Vegetable Intake and Mortality: Results From 2 Prospective Cohort Studies of US Men and Women and a Meta-Analysis of 26 Cohort Studies. Circulation. 2021; Mar 1. doi: 10.1161/CIRCULATIONAHA. 120.048996.

10. AECOSAN - Agencia Española de Consumo Seguridad Alimentaria y Nutrición. Encuesta ENALIA. Encuesta Nacional de Alimentación en la población Infantil y Adolescente. Disponible en: (aesan.gob.es/AECOSAN/web/seguridad_alimentaria/subdetalle/enalia.htm).

11. AECOSAN - Agencia Española de Consumo Seguridad Alimentaria y Nutrición. Encuesta ENALIA 2. Encuesta Nacional de Alimentación en población adulta, mayores y embarazadas. Disponible en: (aesan.gob.es/AECOSAN/web/seguridad_alimentaria/subdetalle/enal ia_2.htm).

12. FĒN 2018. Informe de estado de situación sobre: Frutas y hortalizas, nutrición y salud en la España del $S$ XXI. Disponible en: (fen.org.es/storage/app/media/imgPublicaciones/informe_frutas_y_ hortalizas_fen_2018-v1.pdf)

13. Cámara M, Sánchez Mata, MC, Torija, ME. Frutas y verduras fuente de salud. Monografía n $n^{0}$ 8. Colección Nutrición y Salud. Servicio de Promoción de la Salud. Instituto de Salud Pública. Consejería de Sanidad y Consumo Comunidad de Madrid. 2003.

14. García-Herrera P, Sánchez-Mata MC, Cámara M, Tardío J, OlmedillaAlonso B. Carotenoid content of wild edible young shoots traditionally consumed in Spain (Asparagus acutifolius L., Humulus lupulus L., Bryonia dioica Jacq. and Tamus communis L.). J Sci Food Agric, 2013; 93:1692-1698. doi: 10.1002/isfa.5952

15. Cámara M, Sánchez-Mata MC, Fernández-Ruiz V, Cámara RM, Manzoor S, Caceres J0. Chapter 11 - Lycopene. A review of chemical and biological activity related to beneficial health effects. Studies in Natural Products Chemistry. 2013; 40:383-426.

16. Cebadera-Miranda L, Morales P, Cáma $\neg$ ra M. Chapter 27. Bioactive compounds in oranges from the Mediterranean climate área. En The Mediterranean Diet: An evidence-based approach. Ed. Victor R. Preedy and Ronald Ross Watson. Academic Press, Elservier, 2020.

17. Sánchez-Mata MC, Tardío J. Eds. Mediterranean Wild Edible Plants.Ethnobotany and Food Composition Tables. Springer. 2016.

18. Ciudad-Mulero M, Matallana-González MC, Cámara M, FernándezRuiz V, Morales P. Antioxidant phytochemicals in pulses and it relation to human health: A Review. Current Pharmaceutical Design. 2020; 26:1880-1897. doi: 10.2174/1381612826666200203130150. 
19. Barcina Angulo, Y. Dieta, nutrientes y COVID-19. Noticiero Real Academia Nacional de Farmacia. Disponible en: (ranf.com/noticia/13806/).

20. Calder PC. Nutrition, immunity and COVID-19. British Medical Journal NPH 2020; 3:1,74-92, e000085. Doi:10.1136/ bminph-2020000085

21. Mrityunjaya M, Pavithra V, Neelam R, Janhavi P, Halami PM, Ravindra PV. Immune-Boosting, Antioxidant and Anti-inflammatory Food Supplements Targeting Pathogenesis of COVID-19. Front Immunol. 2020; Oct 7;11:570122. doi: 10.3389/fimmu.2020.570122.

22. Cámara M, Sánchez-Mata MC, Fernández-Ruiz V, Cámara RM, Cebadera E, Domínguez L. A review of the role of micronutrients and bioactive compounds on immune system improvement to fight against the COVID-19 disease. Foods 2021; 10, 1088. D0l: https://doi.org/ $10.3390 /$ foods 10051088

23. Wu D, Lewis ED, Pae M, Meydani SN. Nutritional modulation of immune function: analysis of evidence, mechanisms, and clinical relevance. Front. Immunol. 2019; 9:3160. doi: 10.3389/fimmu.2018.03160

24. Bae M, Kim H. Mini-Review on the Roles of Vitamin C, Vitamin D, and Selenium in the Immune System against COVID-19. Molecules 2020; 25,22:5346. doi: 10.3390/molecules25225346

25. Ku Kumar R, Rathi H, Haq A, Wimalawansa SJ, Sharma A. Putative roles of vitamin $D$ in modulating immune response and immunopathology associated with COVID-19. Virus Res. 2021; 292:198235. doi:10.1016/i.virusres.2020.198235

26. Iddir M, Brito A, Dingeo G, Fernandez Del Campo SS, Samouda H, La Frano MR, Bohn T. Strengthening the Immune System and Reducing Inflammation and Oxidative Stress through Diet and Nutrition: Considerations during the COVID-19 Crisis. Nutrients 2020; 12,6:1562. Doi:10.3390/nu12061562.

27. Shakoor H, Feehan J, Al Dhaheri AS, Ali HI, Platat C, Ismail LC, Apostolopoulos V, Stojanouska L. Immune-boosting role of vitamins D, C, E, zinc, selenium and omega-3 fatty acids: Could they help against COVID-19? Maturitas 2021; 143:1-9. Doi:10.1016/i.maturitas. 2020.08.003

28. Laird, E.; Rhodes, J.; Kenny, R.A. Vitamin D and Inflammation: Potential Implications for Severity of Covid-19. Ir. Med. J. 2020, 113(5), 81. PMID: 32603576.

29. NCT04810949. Effect of Vitamin D Supplementation on the Development of Respiratory Infections (COVID-19) in Health Personnel at Hospital Clínica NOVA With Serum Values > 20ng / ml. https://clinicaltrials.gov/ct2/show/study/NCT04810949?term = vitamin + D\&rec is $=$ eh\&type $=$ Intr\&cond $=$ Covid $19 \&$ draw $=3 \&$ rank $=5$

30. European Parliament and Council of the European Union. Regula- tion (EC) No 1925/2006 of the European Parliament and of the Council of 20 December 2006 on the addition of vitamins and minerals and of certain other substances to foods. Off. J. Eur. Union 2006, L404/26. Disponible en: (eur-lex.europa.eu/legalcontent/EN/ALL/?uri= celex:32006R1925).

31. Domínguez Díaz L, Fernández-Ruiz V, Cámara M. An international regulatory review of food health-related claims in functional food products labeling. J. Funct. Foods 2020, 68:103896. Doi:10.1016/i.fff.2020.103896

32. EU Register of nutrition and health claims made on foods. Disponible en:

(ec.europa.eu/food/safety/labelling nutrition/claims/register/public/?event=register.home).

33. AESAN Agencia Española de Seguridad Alimentaria y Nutrición. Informe del Comité Científico de la Agencia Española de Seguridad Alimentaria y Nutrición sobre Ingestas Nutricionales de Referencia para la población española. Revista del Comité Científico de la AESAN, 2019, 29: 43-68.

34. European Parliament and Council of the European Union. Regulation (EU) No 1169/2011 of the European Parliament and of the Council of 250 ctober 2011 on the provision of food information to consumers, amending Regulations (EC) No 1924/2006 and (EC) 1925/2006 of the European Parliament and of the Council, and repealing Commission Directive 87/250/EEC, Council Directive 90/496/EEC, Commission Directive 1999/10/EC, Directive 2000/13/EC of the European Parliament and of the Council, Commission Directives 2002/67/EC and 2008/5/EC and Commission Regulation (EC) No 608/2004. Off. J. Eur. Union 2011, L304/18. Disponible en: (eur-lex.europa.eu/legalcontent/EN/TXT/?uri=(ELEX:32011R1169).

35. BEDCA. Base de Datos Española de Composición de Alimentos. Red BEDCA y AESAN. Disponible en: (bedca.net).

36. Domínguez Díaz L, Fernández-Ruiz V, Cámara M. The frontier between nutrition and pharma: The international regulatory framework of functional foods, food supplements and nutraceuticals. Crit. Rev. Food Sci. Nutr 2019, 60(10), 1738-1746.

Doi:10.1080/10408398.2019.1592107.

37. European Commission. Action plan on online offers and advertising of food related to COVID-19. Disponible en:

(ec.europa.eu/food/safety/official_controls/eu-coordinated-controlplans/covid-19 en).

38. WHO. Coronavirus disease (COVID-19): Food safety and nutrition. Disponible en: (who.int/news-room/q-a-detail/coronavirus-diseasecovid-19-food-safety-and-nutrition).

39. ISIN. International Society of Immunonutrition. Position Statement on Nutrition, Immunity and COVID-19. Disponible en: (immunonu- 
trition-isin.org/docs/isinComunicadoCovid19.pdf).

40. Tarazona-Santabalbina FJ, Cuadra L, Cancio JM, Roca Carbonell F, Pérez-Castejón Garrote JM, Casas-Herrero A, Martínez-Velilla N, SerraRexach JA, Formiga F. Vitamin D supplementation for the prevention and treatment of COVID-19: a position statement from the Spanish Society of Geriatrics and Gerontology. Revista Española de Geriatría y Gerontología, 2021. Doi:10.1016/i.regg.2021.02.001.

41. EFSA European Food Safety Authority. Dietary Reference Values for nutrients Summary report. 2017. Disponible en: (efsa.onlinelibrary.wiley.com/doi/epdf/10.2903/sp.efsa.2017.e15121).

42. HLPE, 2014. Las pérdidas y el desperdicio de alimentos en el contexto de sistemas alimentarios sostenibles. Un informe del Grupo de alto nivel de expertos en seguridad alimentaria y nutrición del Comité de Seguridad Alimentaria Mundial. Roma, 2014. Disponible en: http://www.fao.org/fileadmin/user_upload/hlpe/hlpe_documents/HL PE_Reports/HLPE-Report-8_ES.pdf

43. ONU. Consumo en tiempos de la COVID-19 Estilos de vida sostenibles en el hogar Disponible en: hitps://wedocs.unep.org/bitstream/handle/20.500.11822/32175/GUIA_CONSUMO_SOSTENIBLE.pdf?sequence $=1$ \&isAllowed $=y$

44. Blay-Palmer A, Carey R, Valette E, Sanderson MR. Post COVID 19 and food pathways to sustainable transformation. Agric Human Values. 2020; May 18:1-3. doi:10.1007/s10460-020-10051-7

45. Willett W, Rockström J, Loken B, Springmann M, Lang T, Vermeulen S, Garnett T, Tilman D, DeClerck F, Wood A, Jonell M, Clark M, Gordon U, Fanzo J, Hawkes C, Zurayk R, Rivera JA, De Vries W, Majele Sibanda L, Afshin A, Chaudhary A, Herrero M, Agustina R, Branca F, Lartey A, Fan S, Crona B, Fox E, Bignet V, Troell M, Lindahl T, Singh S, Cornell SE, Srinath Reddy K, Narain S, Nishtar S, Murray CJL. Food in the Anthropocene: the EAT-Lancet Commission on healthy diets from sustainable food systems. Lancet. 2019 Feb 2;393(10170):447492. doi: 10.1016/50140-6736(18)31788-4.

46. Global Burden of Disease 2020. Disponible en: (thelancet.com/gbd).

47. Derbyshire EJ. Flexitarian Diets and Health: A Review of the Evidence - Based Literature. Front Nutr. 2017, 3:55. doi:10.3389/fnut.2016.00055

48. FAO 2021 Año internacional de la frutas y verduras. Disponible en: (fa0.org/fruits-vegetables-2021/es/).

49. WHO 2017. Ambition and Action in Nutrition 2016-2025. Disponible en: (fao.org/3/CAl435EN/cal435en.pdf).

50. Comisión Europea. FO0D 2030: la investigación innovadora de la Unión Europea garantiza que el sistema alimentario esté preparado para el futuro. Disponible en: (cordis.europa.eu/article/id/400948food-2030-innovative-eu-research-ensures-food-system-is-futureready/es).

Si desea citar nuestro artículo:

Alimentación saludable y sostenible en tiempos

de la pandemia covid-19

Montaña Cámara Hurtado An Real Acad Farm [Internet]. An. Real Acad. Farm. Vol. 87. № 2 (2021) · pp. 195- 204

DOl: http:// 